\title{
artigo
}

Abreu, M.S.; Siqueira, J.,M.M.T.; Maia, J.C.S.; Neponceno, D.B.; Luz, E.B.A.L.; Mendes-Sousa, A.F.

Aspectos epidemiológicos e distribuição espacial da leishmaniose visceral em Picos, Piauí, Brasil

\section{Aspectos epidemiológicos e distribuição espacial da leishmaniose visceral em Picos, Piauí, Brasil}

\author{
Epidemiological aspects and spatial distribution of visceral leishmaniasis in Picos, Piauí, Brazil \\ Aspectos epidemiológicos y distribución espacial de la leishmaniasis visceral en Picos, Piauí, Brasil
}

\begin{abstract}
RESUMO
Objetivo: Objetivou-se investigar o perfil epidemiológico e a distribuição espacial da leishmaniose visceral (LV) em Picos. Métodos: Dados de casos de LV de 2007 a 2019 foram obtidos de registros do Sistema de Informação de Agravos de Notificação através da Secretaria Municipal de Saúde. Resultados: Um total de 64 casos foram confirmados. A LV ocorreu em todos os anos, exceto em 2018. A taxa de incidência média foi de 6,6/100 mil habitantes e a taxa de letalidade média foi de 14,1\%. A maioria dos casos ocorreu em crianças e adultos do sexo masculino, de cor parda e residentes na área urbana. Coinfecção com HIV ocorreu em $6,3 \%$ dos casos. A doença foi confirmada em 14 bairros do município. Conclusão: 0 estudo ratifica a importância da vigilância para LV em áreas do interior do estado, para detecção de mudanças no perfil epidemiológico da LV bem como estabelecer medidas de controle apropriadas.
\end{abstract}

DESCRITORES: Leishmaniose visceral; Epidemiologia; Brasil.

\section{ABSTRACT}

Objective: This study aimed to investigate demographic characteristics and spatial distribution of visceral leishmaniasis (VL) in Picos. Methods: Data of VL cases from 2007 to 2019 were obtained from reports forms from the Brazilian National Information System for Notifiable Diseases, through the Municipal Health Secretary. Results: A total of 64 cases were confirmed. The disease occurred in all studied years, except for 2018. The mean incidence rate was 6.6/100,000 inhabitants. The mean lethality rate was $14.1 \%$. Most of the cases occurred in males, children, people of brown ethnicity and residents in the urban area. HIV-coinfection occurred in $6.3 \%$ of the cases. The disease was reported in 14 neighborhoods of the municipality. Conclusion: Thus, our study supports the relevance of surveillance for $V L$ in the inland areas from the state, in order to pursue changes is the epidemiological profile of the disease and to establish appropriate control measures.

DESCRIPTORS: Visceral leishmaniasis; Epidemiology; Brazil.

\section{RESUMEN}

Objetivo: Éste estudio tuvo como objetivo investigar las características demográficas y la distribución espacial de leishmaniasis visceral (LV) en Picos. Métodos: Los datos de casos confirmados de LV de 2007 a 2019 en Picos se obtuvieron de los registros del Sistema de Información de Enfermedades de Notificación a través de la Secretaría de Salud Municipal. Resultados: Se confirmaron un total de 64 casos. La enfermedad se presentó en todos los años estudiados, excepto en 2018. La tasa de incidencia media fue de 6,6/100.000 personas. La tasa de mortalidad media fue de $14,1 \%$. La mayoría de los casos ocurrieron en niños y adultos del sexo masculino, de color marrón y residentes en el área urbana. La coinfección por VIH en el 6,3\% de los casos. La enfermedad se confirmó en 14 barrios del municipio. Conclusión: Así, nuestro estudio confirma la importancia de la vigilancia de LV en zonas del interior del estado, para detectar posibles cambios en el perfil epidemiológico de la enfermedad así como para establecer las medidas de control adecuadas.

DESCRIPTORES: Leishmaniasis visceral; Epidemiología; Brasil.

RECEBIDO EM: 30/01/2021 APROVADO EM: 11/02/2021

\section{Magna de Souza Abreu}

Acadêmica de Medicina na Universidade Federal do Piauí, Campus Senador Helvídio Nunes de Barros - Picos, PI.

ORCID: 0000-0002-3728-7020 


\section{José Matheus Macedo Torquato de Siqueira}

Acadêmico de Medicina na Universidade Federal do Piauí, Campus Senador Helvídio Nunes de Barros - Picos, PI.

ORCID: 0000-0001-5996-5315

\section{José Cleves da Silva Maia}

Biólogo (UFPI/CSHNB), Mestrando em Ciência Animal na Universidade Federal do Vale do São Francisco - Petrolina, PE. ORCID: 0000-0003-0934-235X

\section{Denise Barguil Nepomuceno}

Biomédica (UNINOVAFAPI), Mestra em Parasitologia (UFMG), Doutora em Parasitologia (UFMG). Atualmente é residente pós-doutoral na Universidade Federal de Minas Gerais - Belo Horizonte, MG.

ORCID: 0000-0003-3314-0491

\section{Ellen Barros Araújo Lopes Luz}

Médica (UPE), Especialista em Pediatria (UFPI), Docente do curso de Medicina na Universidade Federal do Piauí, Campus Senador Helvídio Nunes de Barros - Picos, PI.

ORCID: 0000-0002-7169-7832

\section{Antonio Ferreira Mendes-Sousa}

Médico Veterinário (UFPI), Mestre em Parasitologia (UFMG), Doutor em Parasitologia (UFMG), Docente dos cursos de Medicina e Enfermagem e membro do Núcleo de Pesquisa em Ciências Médicas na Universidade Federal do Piauí, Campus Senador Helvídio Nunes de Barros - Picos, PI.

ORCID: 0000-0002-2019-1348

\section{INTRODUÇÃO}

A leishmaniose visceral (LV), também conhecida como calazar, é uma doença tropical crônica considerada um problema de saúde pública em mais de 80 países e afeta principalmente áreas de pobreza na Ásia, África, América do Sul e região do Mediterrâneo ${ }^{1,2}$. Na América Latina, mais de $90 \%$ dos casos ocorrem no Brasil, com registros de 4.200 a 6.300 novos casos anuais ${ }^{3,4}$. No Brasil, a LV é causada pelo protozoário Leishmania infantum, transmitida por flebotomíneos do gênero Lutzomyia e tem os cães domésticos como principais reservatórios do parasito $^{3,5}$. A doença é caracterizada por febre prolongada, perda de peso, astenia, hepatoesplenomegalia e anemia, evoluindo para o óbito se não tratada ${ }^{6}$.

Inicialmente rural, a LV tem se disseminado amplamente pelo país, ocorrendo atualmente em 21 das 27 unidades federais brasileiras ${ }^{6}$. A urbanização da LV tem sido descrita desde a década de $80 \mathrm{em}$ grandes cidades como Teresina e Belo Horizonte $^{7,8}$, tornando-se um grave problema de saúde pública por ocorrer em epidemias urbanas e pela possibilidade de evoluir para formas graves que requerem in-
No Brasil, a LV

é causada pelo protozoário

Leishmania

infantum,

transmitida por

flebotomíneos

do gênero

Lutzomyia e tem

os cães domésticos

como principais

reservatórios do

parasito. ternação hospitalar, com elevadas taxas de mortalidade e letalidade, principalmente em crianças e pessoas com comorbidades. A LV é uma doença de notificação compulsória e nos últimos anos manteve incidência de 2 casos/100.000 habitantes no país, porém a taxa de letalidade aumentou de 3,1\% em 2000 para 7,1\% em 20189, exigindo ações de vigilância e controle nas áreas endêmicas.

No estado do Piauí a LV é endêmica, com os primeiros casos relatados em 193410. Teresina, sua capital, registrou mais de mil casos da doença entre 1981 e 1986 , sendo esta a primeira grande epidemia urbana de LV no Brasil ${ }^{11}$. Além disso, entre 1999 e 2009, o Piauí registrou 2.498 casos da doença, sendo 1.369 na sua capital Teresina, o que levou à realização de vários estudos sobre seus aspectos ecoepidemiológicos ${ }^{11,13-15}$.

Apesar de endemicidade da LV no estado, dados sobre a ocorrência da doença fora da capital são escassos e imprecisos. Em Picos, terceira cidade mais populosa e importante centro econômico do estado, a LV é registrada desde a década de 197010 mas a dinâmica da sua ocorrência e distribuição ainda não é bem conhecida. $\mathrm{O}$ município está localizado na região sudeste 
do estado do Piauí, a $315 \mathrm{~km}$ da capital, pertencendo ao bioma caatinga com clima tropical semiárido, média pluviométrica anual de 795,4 mm e temperatura média de $28^{\circ} \mathrm{C}^{16,17}$. Sua população estimada é 78.222 habitantes, sendo $80 \%$ residentes em área urbana ${ }^{16}$. Além disso, possui o segundo maior entroncamento rodoviário da região Nordeste, com intenso fluxo de pessoas provenientes de áreas endêmicas para LV, como a capital Teresina e os estados vizinhos Ceará e Pernambuco ${ }^{8,18,19}$.

Assim, este estudo objetivou investigar a situação da leishmaniose visceral em Picos, Piauí, através da avaliação das suas características epidemiológicas e distribuição espacial.

\section{MÉTODOS}

Trata-se de um estudo de caráter quali-quantitativo baseado em análise epidemiológica descritiva retrospectiva utilizando dados registrados nas fichas de leishmaniose visceral do Sistema de Informação de Agravos de Notificação (SINAN). Os dados de casos de LV registrados de janeiro de 2007 a dezembro de 2019 em Picos foram obtidos do SINAN através do Departamento de Vigilância Epidemiológica da Secretaria Municipal de Saúde. Todos os casos confirmados de LV em indivíduos residentes em Picos foram incluídos. Casos de LV diagnosti-

Figura 1: Número de casos, taxa de incidência e taxa de letalidade da leishmaniose visceral em Picos, Piauí, Brasil, de 2007 a 2019.

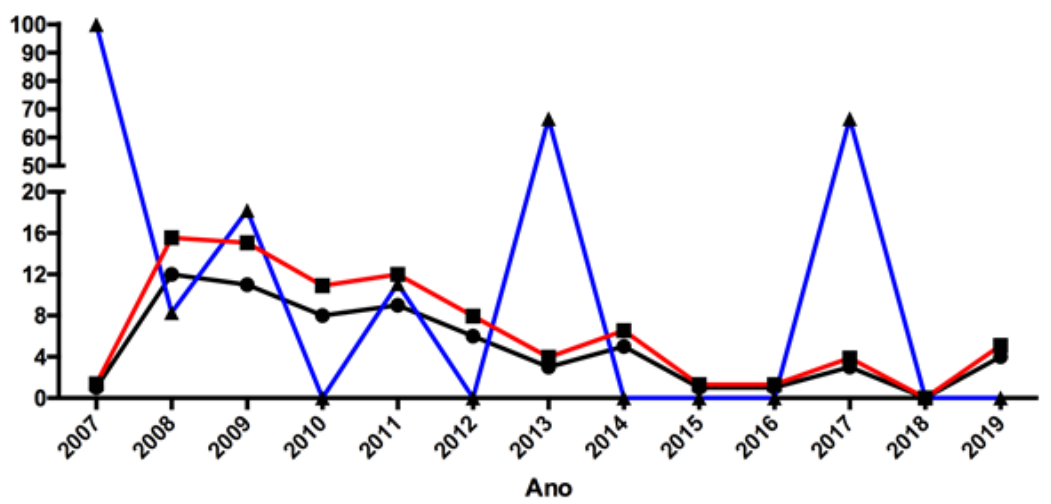

Número de casos (n) Taxa de incidência por 100.000 cados em Picos em residentes de outros municípios foram excluídos da pesquisa.

Os dados foram obtidos de maio a julho de 2020 e armazenados em planilha do programa Microsoft Excel versão 2016 para organização e análise. Frequências relativas foram calculadas para as seguintes variáveis: idade, sexo, cor da pele, zona e coinfecção com vírus da imunodeficiência humana (HIV). Taxas de incidência foram calculadas para 100.000 habitantes. A taxa de letalidade foi calculada dividindo o número de mortes anuais por LV pelo número de casos confirmados no respectivo ano e então multiplicado por 100. Dados da estimativa da população anual foram obtidos do Instituto Brasileiro de Geografia e Estatística - IBGE. A construção do gráfico de indicadores e do mapa de distribuição espacial foi realizada, respectivamente, com os softwares GraphPad Prism 6 e QGIS 3.10. O estudo foi aprovado pelo Comitê de Ética em Pesquisa da Universidade Federal do Piauí com número de protocolo 3.579.481 (CAAE 20387019.6.0000.8057).

\section{RESULTADOS}

De 2007 a 2019, 120 casos de LV foram notificados em Picos, dos quais 64 foram confirmados. A média anual foi de 4,9 casos. Em todos os anos estudados houve pelo menos um caso registrado da doença, exceto pelo ano 2018 (Figura 1). O maior número de casos ocorreu em 2008, com 12 casos confirmados, seguido por 2009 e 2011 com 11 e 9 casos, respectivamente. Houve um declínio de casos nos anos subsequentes, até 0 caso em 2018. A taxa de incidência aumentou de 1,38 em 2007 para 16,65 em 2008 e em seguida diminuiu ao longo dos anos para 0,0 em 2018 (Figura 1). Um aumento na incidência para 5,11 foi observado em 2019, que apresentou 4 novos casos. A incidência média foi de 6,6 casos por cem mil habitantes. Nove mortes por LV foram registradas, com taxa de letalidade média de 14,1\% (Figura 1).

A maioria dos casos ocorreu em indivíduos do sexo masculino $(59,4 \%, \mathrm{n}=38)$. A idade dos pacientes variou de 4 meses 
Tabela 1: Leishmaniose visceral em Picos, Piauí, Brasil, de acordo com sexo, idade, cor da pele, zona e coinfecção com HIV de 2007 a 2019.

$$
\text { VARIÁVEL }
$$

\section{Sexo}

Feminino

Masculino

Idade

$0-10$ anos

$21-30$ anos

$31-40$ anos

$41-50$ anos

$51-60$ anos

$>60$ anos

Cor da pele

Preta

Amarela

Branca

Ignorado

Zona

Rural

Urbana

Coinfecção com HIV

Não

Sim

Ignorado

\section{$\mathrm{N}$}

26

38

40,6

5

11$$
4
$$

$\%$

59,4

40,6

7,8

17,2

15,6

4,7

3,1

10,9

79,7

1,6

6,3

6,3

71,9

45

81,3

4

6,3

8
12,5
Figura 2: Distribuição espacial dos casos de leishmaniose visceral na área urbana de Picos, Piauí, Brasil, de 2007 a 2019

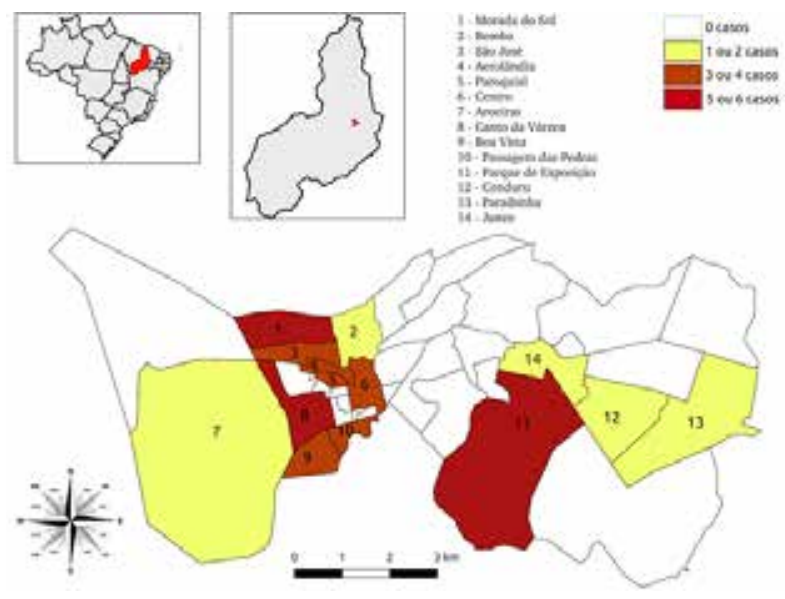

Fonte: SINAN, 2020 a 87 anos. Crianças de 0 a 10 anos foram as mais afetadas $(40,6 \%, n=26)$, seguidas de adultos de 21 a 31 anos $(17,2 \%, n=11)$ e 31 a 40 anos $(15,6 \%, n=10)$ (Tabela 1). A doença predominou na zona urbana $(71,9 \%, \mathrm{n}=45)$, e a maioria dos casos $(79,7 \%)$ foi registrada em pessoas pardas. A coinfecção Leishmania-HIV foi registrada em 6,3\% ( $\mathrm{n}=4)$ dos indivíduos (Tabela 1). Esta informação foi ignorada em $12,5 \%(\mathrm{n}=8)$ dos casos.

A leishmaniose visceral foi confirmada em 14 bairros da zona urbana de Picos (Figura 2). O maior número de casos foi registrado no bairro Parque de Exposição $(n=6)$, seguido dos bairros Morada do Sol e Canto da Várzea, com 5 casos. Os bairros Aerolândia, Boa Vista, Centro e Passagem das Pedras apresentaram 4 casos. Já os bairros São José e Paroquial registraram 3 casos, enquanto Paraibinha e Aroeira, 2 casos. Um caso foi confirmado nos bairros Conduru, Bomba e Junco.

\section{DISCUSSÃO}

Um surto de LV foi observado em Picos no ano de 2008, com aumento acentuado na taxa de incidência. Em seguida, um declínio foi observado até o ano de 2019, seguindo a tendência decrescente de casos de LV no Brasil e no Piauí após o ano $2000^{4,2} 1$. De acordo com a classificação do Ministério da Saúde, o município passou de área de transmissão intensa de LV (média de casos nos últimos 3 anos $>4,4)$ para área de transmissão esporádica (média de casos nos últimos 3 anos $>0$ e $<2,4)$ nos anos recentes ${ }^{6}$. Entretanto, após um grande declínio de 2008 a 2018, a incidência de LV em Picos tornou a aumentar em 2019, ratificando a importância da manutenção da vigilância epidemiológica e medidas de controle no município para evitar comportamento cíclico da LV como tem ocorrido em outras áreas, com surtos em intervalos periódicos ${ }^{11,21-23}$.

Houve diminuição também na taxa de letalidade para 0\% em 2019. Entretanto, a letalidade média por LV no período avaliado permaneceu mais alta que média nacional de 6,9\%9. Taxas elevadas de mor- 
talidade podem ser devido a serviços de saúde deficientes, levando a diagnóstico e tratamento tardios dos pacientes ${ }^{24}$. A toxicidade do tratamento, coinfecções e imunossupressão também podem favorecer ao aumento da taxa de letalidade ${ }^{24}$.

A LV predominou em homens e em crianças, seguindo o padrão da doença no Brasil $^{20-23}$. A incidência maior em homens pode ter causa comportamental, pois homens são mais envolvidos em atividades noturnas fora do domicílio, estando mais expostos ao vetor, além de causa fisiológica, pois níveis elevados de testosterona estimulam resposta imune anti-inflamatória do tipo Th2, tornando-os mais susceptíveis à Leishmania ${ }^{25}$. A alta incidência observada em adultos confirma mudanças descritas na epidemiologia da LV no Brasil, com casos crescentes em adultos ${ }^{20,21}$. Crianças são sabidamente mais susceptíveis ao parasito devido à imaturidade do sistema imune.

Entre os casos confirmados, 6,3\% estavam infectados com HIV, valor inferior às frequências relativas de coinfecção no Brasil $(8,5 \%)^{26}$. Isto pode ser explicado pelo tamanho médio de Picos, uma vez que o HIV é mais prevalente em grandes cidades $^{27}$. Entretanto, como em 12,5\% dos casos o status de HIV foi ignorado, é possível que a proporção de coinfectados seja maior. Além disso, a maioria dos casos ocorreu em pessoas pardas, como relatado em outros municípios nordestinos ${ }^{28}$.

Os casos de LV predominaram na zona urbana, concordando com o processo de urbanização da doença no Brasil ${ }^{7}$. De fato, o vetor Lutzomyia longipalpis já foi capturado em casas da zona urbana de $\mathrm{Picos}^{29}$. O município está localizado em bioma que favorece o desenvolvimento do vetor, além de possuir muitos morros e ser cortado pelo Rio Guaribas, criando áreas favoráveis para a transmissão de Leishmania (ex.: pés de morros e margens de rio) ${ }^{8}$. O bairro com maior número de casos (Parque de Exposição) está localizado na periferia da cidade e é caracterizado por condições socioeconômicas desfavoráveis, como saneamento básico e coleta de lixo inadequados ou inexistentes, fatores associados à alta incidência de $L V^{15}$. Entretanto, a maioria dos bairros com elevado número de casos está localizada na área central da cidade, com características urbanas, como ruas asfaltadas, luz elétrica e água encanada. Porém, esses bairros apresentam áreas de concentração de pobreza e alguns estão localizados em pés de morros, favorecendo o desenvolvimento do vetor e a transmissão do parasito. $\mathrm{O}$ mapa de distribuição espacial pode auxiliar as instituições munici-

\section{A incidência}

maior em homens

pode ter causa

comportamental,

pois homens são

mais envolvidos em

atividades noturnas

fora do domicílio,

estando mais expostos

ao vetor, além de

causa fisiológica,

pois níveis elevados

de testosterona

estimulam resposta

imune anti-

inflamatória do tipo

Th2, tornando-os

mais susceptíveis à

Leishmania pais de saúde a identificar áreas de prioridade para vigilância e controle da LV.

O presente estudo utilizou dados secundários dos formulários de leishmaniose visceral do SINAN, constituindo uma limitação devido ao possível preenchimento incompleto pelos profissionais de saúde, o que dificulta análises mais profundas da epidemiologia da LV em Picos. Além disso, é possível que a doença seja subnotificada em Picos devido às limitações de diagnóstico laboratorial no município, como relatado em outras cidades brasileiras ${ }^{19}$. Contudo, como a LV é uma doença de notificação compulsória no Brasil desde a década de noventa, sabidamente endêmica em Picos e o seu tratamento específico é fornecido apenas pelo Sistema Único de Saúde, assumimos que tais possíveis falhas sejam mínimas e que os dados obtidos no estudo representam a maioria dos casos ocorridos durante o período determinado, representando portanto a realidade da ocorrência da doença do município. Assim, nosso estudo apresenta pela primeira vez uma visão geral dos aspectos epidemiológicos e distribuição espacial da leishmaniose visceral em Picos, um importante polo comercial e área endêmica no estado do Piauí, podendo amparar medidas de prevenção e controle da doença no estado.

\section{CONCLUSÃO}

A leishmaniose visceral é endêmica e bem distribuída na zona urbana de Picos, com taxas de incidência e letalidade decrescentes nos últimos anos mas ainda acima da média nacional. A doença ocorre predominantemente em crianças de até 10 anos e em adultos do sexo masculino, de cor parda e residentes na zona urbana. Nosso estudo reforça a importância da vigilância epidemiológica para a LV em Picos e a necessidade de capacitação dos médicos da Estratégia Saúde da Família para diagnóstico e tratamento precoces da doença, a fim de evitar aumento na incidência da doença e evolução desfavorável dos pacientes. Além disso, o conhecimento da distribuição espacial dos casos no municí- 
pio serve de base para o direcionamento de estratégias de prevenção pelas autori- dades de saúde para áreas prioritárias de risco, diminuindo custos e aumentando potencialmente sua eficácia no combate à LV em Picos. -

\section{REFERÊNCIAS}

1. Reis LL, Balieiro AAS, Fonseca FR, Gonçalves MJF. Changes in the epidemiology of visceral leishmaniasis in Brazil from 2001 to 2014. Rev Soc Bras Med Trop. 2017;50:638-45.

2. World Health Organization (WHO). Leishmaniasis. Key Facts. [internet]. 2020. Available from: https://www.who.int/news-room/ fact-sheets/detail/leishmaniasis.

3. Alvar J, Vélez ID, Bern C, Herrero M, Desjeux P, Cano J, et al. Leishmaniasis worldwide and global estimates of its incidence. PLoS One. 2012;7:e35671.

4. Bezerra JM, Araújo VE, Barbosa DS, Martins-Melo FR, Werneck $\mathrm{GL}$, et al. Burden of leishmaniasis in Brazil and federated units, 1990-2016: Findings from Global Burden of Disease Study 2016. PLoS Negl Trop Dis. 2018;12:e0006697.

5. Lainson R, Rangel EF. Lutzomyia longipalpis and the eco-epidemiology of American visceral leishmaniasis, with particular reference to Brazil - A Review. Mem Inst Oswaldo Cruz. 2005;100:811-27.

6. Ministério da Saúde (MS). Secretaria de Vigilância em Saúde. Guia de Vigilância em Saúde. 1ª edição atualizada. Brasília: MS; 2017. $812 \mathrm{p}$.

7. Harhay MO, Olliaro PL, Costa DL, Costa CHN. Urban parasitology: visceral leishmaniasis in Brazil. Trends Parasitol. 2011;27:403-9.

8. Costa CHN. Characterization and speculations on the urbanization of visceral leishmaniasis in Brazil. Cad Saúde Pública. 2008;24:2959-63.

9. Ministério da Saúde (MS). Secretaria de Vigilância em Saúde. Boletim Epidemiológico Panorama da vigilância de doenças crônicas não transmissíveis no Brasil. Volume 50. Brasilia: MS; 2019. 15 p.

10. Costa CHN, Pereira HF, Araújo M V. Epidemia de leishmaniose visceral no estado do Piauí, Brasil, 1980-1986. Rev Saúde Pública. 1990;24:361-72.

11. Werneck GL, Pereira TJC, Farias GC, Silva FO, Chaves FC, et al. Avaliação da efetividade das estratégias de controle da leishmaniose visceral na cidade de Teresina, Estado do Piauí, Brasil: resultados do inquérito inicial - 2004*. Epidemiol e Serviços Saúde. 2008;17:87-96.

12. Drumond O, Costa FAL. Forty years of visceral leishmaniasis in the state of Piaui: a review. Rev Inst Med Trop Sao Paulo. 2011;53:3-11.

13. Werneck GL, Costa CHN, Walker AM, David JR, Wand M, Maguire $\mathrm{JH}$. The Urban Spread of Visceral Leishmaniasis: Clues from Spatial Analysis. Epidemiology. 2002;13:364-7.

14. Werneck GL, Costa CHN, Walker A, David JR, Wand M, Maguire $\mathrm{JH}$. Multilevel modelling of the incidence of visceral leishmaniasis in Teresina, Brazil. Epidemiol Infect. 2006;135:195-201.

15. Cerbino Neto J, Werneck GL, Costa CHN. Factors associated with the incidence of urban visceral leishmaniasis: an ecological study in Teresina, Piauí State, Brazil. Cad Saúde Pública. 2009;25:1543-51.
16. Instituto Brasileiro de Geografia e Estatística (IBGE). Picos. [internet]. 2019. Available from: https://cidades.ibge.gov.br/brasil/pi/picos/panorama

17. Instituto Nacional de Metereologia (INMET). [internet] 2019. Available in: http://www.inmet.gov.br/portal/.

18. Cavalcante İM, Vale MR. Epidemiological aspects of visceral leishmaniasis (kala-azar) in Ceará in the period 2007 to 2011. Rev Bras Epidemiol. 2014;17:911-24.

19. Araujo AC, Gonçalves NNVM, Dantas-Torres F, Ferreira F, Horta MC. Visceral leishmaniasis in Petrolina, state of Pernambuco, Brazil, 2007-2013. Rev Inst Med Trop Sao Paulo. 2016;58:1-4.

20. Lima ID, Lima ALM, Mendes-Aguiar CO, Coutinho JFV, Wilson $\mathrm{ME}$, Pearson RD, et al. Changing demographics of visceral leishmaniasis in northeast Brazil: Lessons for the future. PLoS Negl Trop Dis. 2018;12:e0006164.

21. Lima ÁLM, de Lima ID, Coutinho JFV, Sousa ÚPST, Rodrigues MAG, Wilson ME, et al. Changing epidemiology of visceral leishmaniasis in northeastern Brazil: A 25-year follow-up of an urban outbreak. Trans R Soc Trop Med Hyg. 2017;111:440-7.

22. Diniz LFB, Souza CDF, Carmo RF. Epidemiology of human visceral leishmaniasis in the urban centers of the lower-middle São Francisco Valley, Brazilian semiarid region. Rev Soc Bras Med Trop. 2018;51:461-6.

23. Cavalcante FRA, Cavalcante KKS, Florencio CMGD, Moreno JO, Correia FGS, Alencar CH. Human visceral leishmaniasis: Epidemiological, temporal and spacial aspects in Northeast Brazil, 2003-2017. Rev Inst Med Trop Sao Paulo. 2020;62:e12.

24. Belo VS, Struchiner CJ, Barbosa DS, Nascimento BWL, Horta MAP, Silva ES, et al. Risk Factors for Adverse Prognosis and Death in American Visceral Leishmaniasis: A Meta-analysis. PLoS NegI Trop Dis. 2014;8: e2982

25. Bernin $\mathrm{H}$, Lotter $\mathrm{H}$. Sex bias in the outcome of human tropical infectious diseases: Influence of steroid hormones. J Infect Dis. 2014;209 Suppl. 3:S107-13.

26. Lindoso JA, Cota GF, Cruz AM, Goto H, Maia-Elkhoury ANS, Romero GAS, et al. Visceral Leishmaniasis and HIV Coinfection in Latin America. PLoS NegI Trop Dis. 2014;8:e3136.

27. Luz PM, Veloso VG, Grinsztejn B. The HIV epidemic in Latin America: Accomplishments and challenges on treatment and prevention. Curr Opin HIV AIDS. 2019;14:366-73.

28. Sousa JMS, Ramalho WM, Melo MA. Demographic and clinical characterization of human visceral leishmaniasis in the State of Pernambuco, Brazil between 2006 and 2015. Rev Soc Bras Med Trop. 2018;51:622-30.

29. Andrade Filho JD, Silva ACL, Falcão AL. Phlebotomine sand flies in the state of Piauí, Brazil (Diptera: Psychodidae: Phlebotominae). Mem Inst Oswaldo Cruz. 2001;96:1085-1087. 